\title{
Impact of vancomycin resistance on mortality in neutropenic patients with enterococcal bloodstream infection: a retrospective study
}

Sung-Yeon Cho ${ }^{1}$, Dong-Gun Lee ${ }^{1,2^{*}}$, Su-Mi Choi ${ }^{1}$, Jae-Cheol Kwon ${ }^{3}$, Si-Hyun Kim, Jae-Ki Choi ${ }^{1}$, Sun Hee Park', Yeon-Joon Park, Jung-Hyun Choi ${ }^{1}$ and Jin-Hong Yoo ${ }^{1}$

\begin{abstract}
Background: Vancomycin-resistant Enterococcus (VRE) bloodstream infection (BSI) is generally associated with the delayed administration of adequate antibiotics. The identification of risk factors and outcomes of VRE BSI is necessary for establishing strategies for managing neutropenic fever in patients with hematological malignancies.

Methods: We retrospectively analysed consecutive cases of enterococcal BSI in patients with neutropenia after chemotherapy or stem cell transplantation between July 2009 and December 2011 at a single center.

Results: During the 30-month period, among 1,587 neutropenic patients, the incidence rate of enterococcal BSI was 1.76 cases per 1,000 person-days. Of the 91 enterococcal BSIs, there were 24 cases of VRE. VRE BSI was associated with $E$. faecium infection $(P<.001)$, prolonged hospitalization $(P=.025)$ and delayed administration ( $\geq 48$ hours after the febrile episode) of adequate antibiotics ( $P=.002$ ). The attributable mortality was $17 \%$ and $9 \%$ for VRE and vancomycin-susceptible Enterococcus (VSE), respectively ( $P=.447)$. The 30-day crude mortality was $27 \%$ and $23 \%$ for VRE and VSE, respectively (OR 1.38,95\% CI 0.53-3.59; $P=.059)$. Only SAPS-II was an independent predictive factor for death (adjusted OR 1.12, 95\% Cl 1.08-1.17; $P<.001$ ).

Conclusions: In conclusion, vancomycin resistance showed some trend towards increasing 30-day mortality, but is not statistically significant despite the delayed use of adequate antibiotics ( $\geq 48$ hours). Only underlying severity of medical condition predicts poor outcome in a relatively homogeneous group of neutropenic patients.
\end{abstract}

Keywords: Bacteremia, Enterococcus, Mortality, Neutropenia, Risk factors, Vancomycin resistance

\section{Background}

Enterococcal bloodstream infections (BSI) have been increasing in hospitalized patients since the 1990s [1,2]. Enterococci are normal intestinal flora of humans, but usually cause infections when immunity of the host is compromised [3]. At our Blood and Marrow Transplantation Center, Enterococcus has emerged as the third most common pathogen, constituting $14.0 \%$ of all BSIs, and vancomycin resistant Enterococcus (VRE) was responsible

\footnotetext{
* Correspondence: symonlee@catholic.ac.kr

'Division of Infectious Diseases, Department of Internal Medicine, Vaccine Bio Research Institute, College of Medicine, The Catholic University of Korea, Seoul, Republic of Korea

${ }^{2}$ The Catholic Blood and Marrow Transplantation Center, Seoul St. Mary's Hospital, College of Medicine, The Catholic University of Korea, Seoul, Republic of Korea

Full list of author information is available at the end of the article
}

for $20.6 \%$ of proven enterococcal BSIs [4]. There are several studies on outcomes after administration of newer antimicrobial agents such as linezolid, daptomycin, quinupristin-dalfopristin or tigecycline $[5,6]$. But VRE BSI is still a major concern, because of the limited therapeutic options available and higher mortality subsequent to infection $[7,8]$.

It has been demonstrated that vancomycin resistance increases mortality in patients with enterococcal BSI [1,9-14]; however, controversy remains [5,15-19]. Conflicting results on the clinical implications of vancomycin resistance may have been due to the varied severity of the underlying illness in patients with VRE BSI. Previous meta-analysis concluded that vancomycin resistance is independently associated with increased mortality in enterococcal BSI [1]. However, only a subset 
of the studies involved in the meta-analysis examined immunocompromised individuals $[13,14]$.

Whether vancomycin resistance increases mortality in patients with enterococcal BSI could be one of the important considerations in determining empirical therapeutic approach, especially in the setting of chemotherapyinduced reversible neutropenia. This study examined the risk factors and outcomes of VRE BSI as compared with vancomycin-susceptible Enterococcus (VSE) BSI, and the factors associated with mortality in a relatively homogeneous group of patients with neutropenia after chemotherapy or stem cell transplantation (SCT) for underlying hematologic diseases.

\section{Methods}

\section{Study design and clinical setting}

We retrospectively reviewed all consecutive episodes of enterococcal BSIs in adult patients with neutropenic fever from July 2009 to December 2011 at the Catholic Blood and Marrow Transplantation Center, Seoul St. Mary's Hospital. Our hospital is a 1,200-bed, universityaffiliated, tertiary-care center in Seoul, South Korea. The Catholic Blood and Marrow Transplantation Center performs over 350 SCTs annually.

Eligible patients included those with hematological malignancies, who experienced neutropenic fever during chemotherapy or SCT, who were older than 18 years of age, and were documented to be blood culture positive for Enterococcus species. Enterococcal BSI cases were obtained from a computerized microbiology database. We collected data targeted to positive blood culture results from chemotherapy ward and SCT ward. In cases with persistent BSI, we analysed the first episode. If cases met the definition of separate BSI, each infection was considered individually. Concomitant VRE and VSE BSI were excluded. Even in cases with neutropenic fever and documented enterococcal BSI, patients who had not previously received treatment for hematological malignancies, such as anti-cancer chemotherapy or SCT, were excluded.

The data obtained for each patient included age, sex, underlying diseases, absolute neutrophil count (ANC) at the onset of fever, severity and duration of neutropenia, severity of illness (Simplified Acute Physiology Score, SAPS II) and Charlson's comorbidity index at the onset of BSI, length of hospital stay, stay in intensive care unit (ICU) before the onset of BSI, septic shock, the presence of previously documented rectal VRE colonization, organisms isolated from blood and antimicrobial susceptibility, antibiotics administered, and survival 7 and 30 days after the onset of BSI.

Blood for cultures was sampled using sterile techniques, with one set from peripheral vein puncture and another set simultaneously from a central venous catheter.
Each 10 15 mL of blood was inoculated into aerobic and anaerobic bottles (BD BACTEC ${ }^{\mathrm{mm}}$ Plus Aerobic/F, Lytic/10 Anaerobic/F Culture Vials, Becton Dickinson, Sparks, MD, USA), which were immediately transported to the clinical laboratory. Antibiotic susceptibility testing was performed with an automatic system (Vitek-2, bioMérieux, Hazelwood, MO, USA). E. faecalis clinical isolates are almost always susceptible to ampicillin; hence, ampicillin sensitivity of E. faecalis was not tested in our study. Polymerase chain reaction (PCR) test to look for van A, B, C genes was not performed routinely.

At our center, during the chemotherapy or SCT, oral ciprofloxacin (500 $\mathrm{mg}$ twice a day) is used as routine prophylaxis. The initial empirical treatment of neutropenic fever in hematological malignancy patients is to use anti-pseudomonal cephalosporin (cefepime or ceftazidime) and/or an aminoglycoside (isepamicin), excluding the initial use of glycopeptides. The use of glycopeptides as empirical antimicrobial therapy is recommended if the patient's blood cultures are positive for Gram-positive bacteria, a catheter-related infection is suspected, there is a history of MRSA infection, the patient has severe sepsis or shock pending the results of cultures, the patient has severe mucositis, or the patient has skin or soft tissue infection. If Gram-positive bacteremia persists for 2 or 3 days after adding glycopeptides, we consider anti-VRE therapy before receiving the final microbiology results $[20,21]$.

Rectal VRE surveillance is not routinely performed in patients undergoing chemotherapy or SCT according to ASBMT guidelines [22]. For cases of previous VRE infection or hospitalization in the ICU, rectal swabs for VRE surveillance are performed. All VRE-colonized or infected persons are placed under contact precautions for nosocomial infection control until they have three consecutive negative results.

The Institutional Review Board of Seoul St. Mary's hospital approved the research protocol with a waiver of informed consent (KC12RISI0314).

\section{Definitions}

Enterococcal BSI was defined as isolation of Enterococcus species in two or more blood culture results $[1,8,13,15,17]$. Enterococcal BSI occurring 60 or more days after a previous initial episode was considered a separate episode $[8,12]$. Neutropenia was defined as an ANC $<500 / \mathrm{mm}^{3}$, or $<1000 / \mathrm{mm}^{3}$ with predicted falls to $<500 / \mathrm{mm}^{3}$ within $2 \sim 3$ days. Severe neutropenia was defined as an ANC $<100 / \mathrm{mm}^{3}[20,23]$. Fever was defined as an increase in body temperature to over $38.0^{\circ} \mathrm{C}$ using a tympanic thermometer or to over $37.5^{\circ} \mathrm{C}$ using an axillary thermometer [20]. The length of hospitalization was defined as the number of days from hospital admission 
to the development of clinically significant enterococcal bacteremia. Intermediate susceptibility was classified as 'non-susceptible'. Clinical outcomes were classified as 'improvement' indicating resolution of the signs of systemic inflammatory response syndrome (SIRS), 'failure' for any sustained signs of infection or death [24]. Microbiologic outcomes were classified as 'eradication' or 'persistence' [11]. When any follow-up blood culture result was positive for Enterococcus species, the case was considered as 'persistence'. When 7-day microbiologic outcome showed 'persistence', it considered as 'microbiologic failure' at 7 day after the febrile episode. Mortality was considered attributable to the Enterococcus if the patient died within 7 days of the BSI and no other cause could be identified. Crude mortality was defined as mortality that occurred within the month that followed a BSI episode [25]. Empirical antimicrobial therapy was considered to be appropriate when at least one active antimicrobial agent susceptible to the organism by in vitro test was administered [26]. The length of delay until adequate antibiotics treatment was defined as the interval between the onset of fever and the time that antibiotics to which the organism was susceptible were administered. Delayed administration of adequate antibiotics was defined when the length of the delay was 48 hours or more [12].

\section{Statistical analysis}

To identify factors associated with the development of VRE BSI, chi-square analysis or Fisher's exact test was used to compare categorical variables, and a Student's $t$-test or Mann-Whitney $U$ test was used to compare continuous variables. In order to identify factors associated with mortality, Cox proportional hazard model with backward method was used to control for the effects of confounding variables. Kaplan-Meier survival curves were used to analyse the mortality trends, and the results were compared using the log-rank test. SAPS-II score at the onset of febrile episode was analysed by using receiver operating characteristic (ROC) curve. For measures of association, a 2-tailed $P<.05$ was considered to be significant. Statistical analysis were performed using SAS software ver. 8.2 (SAS Institute Inc., Cary, NC, USA).

\section{Results}

During the 30-month period, among a total of 2,028 patients admitted with hematological malignancies, 1,587 developed neutropenia during chemotherapy or SCT, and 91 episodes of enterococcal BSIs were identified. Of these infectious episodes, 24 (26.4\%) were VRE BSIs. The incidence rate of enterococcal BSI in neutropenic patients who underwent chemotherapy or SCT was 1.76 cases per 1,000 person-days. There were 2 cases of concurrent VRE and VSE bacteremia. It was excluded because of the interpretative problem. A review of monthly incidence rates of enterococcal BSIs showed that no outbreaks occurred during this study period.

The baseline characteristics are shown in Table 1. There were no differences in age, sex, SAPS-II and Charlson's comorbidity index, and the duration and severity of neutropenia between VSE and VRE BSI groups. VRE BSI was associated with $E$. faecium infection $(P<.001)$ and prolonged hospitalization $(P=.025)$. All of the patients in this study had a central venous catheter for their chemotherapy or SCT, which was Hickman catheter or chemoport. Delayed administration of adequate antibiotics (more than 48 hours after the febrile episode) was more common in VRE BSI than in the VSE BSI group $(P=.002)$. The median interval from the onset of the febrile episode to the administration of adequate antibiotics was 1.4 (range $<1 \sim 5$ ) days in the VSE BSI group and 2.8 (range $<1 \sim 5$ ) days in the VRE BSI group. Enterococcal BSI developed a median of 14 days after the onset of neutropenia. In the VSE BSI group, $61.2 \%$ of the patients had acute myeloid leukaemia (AML), while acute lymphoblastic leukaemia (ALL) was the major underlying disease $(54.2 \%)$ in the VRE BSI group $(P=.046)$.

The outcomes are shown in Table 2. The 7-day clinical treatment failure rate was $50.0 \%$ and $26.9 \%$ in VRE and VSE BSI, respectively $(P=.040)$, while microbiologic failure at 7 days after the onset of BSI did not differ significantly $(16.7 \%$ and $11.9 \%, P=.499)$. Despite the delayed administration of adequate antibiotics and delayed resolution of the signs of SIRS in the VRE BSI group, the attributable mortality was $16.7 \%$ for VRE and $9.0 \%$ for VSE BSI $(P=.447)$. The 30-day crude mortality was $27.3 \%$ for VRE and $22.7 \%$ for VSE (hazard ratio [HR] $1.38,95 \%$ confidence interval $[\mathrm{CI}] 0.53-3.59 ; P=.059)$. Kaplan-Meier curves comparing patient survival are shown in Figure 1. In the Cox proportional hazard analysis, with the satisfaction of the assumption of 'proportional hazards', only SAPS-II at the onset of BSI proved to be an independent risk factor for death (adjusted HR 1.12, 95\% CI 1.08-1.17; $P<.001$ ) (Table 3). Analysing by ROC curve, SAPS-II at the onset of febrile episode is a good predictive factor for 30-day crude mortality with area under the curve (AUC) value of $0.817(P<.001)$. With a cut-off value of 42.5 , the sensitivity and specificity for 30-day crude mortality was 0.810 and 0.791 , respectively.

Table 4 shows the susceptibility patterns according to Enterococcus species. Of the 91 isolates, 66 (72.5\%) were E. faecium, 23 (25.3\%) were E. faecalis, and 2 (2.2\%) were E. gallinarum. One-third of the E. faecium isolates (22 of 66) were resistant to vancomycin. Both E. gallinarum isolates were resistant to vancomycin. Of the 
Table 1 Descriptive baseline characteristics of patients with enterococcal bloodstream infections

\begin{tabular}{|c|c|c|c|}
\hline & VSE BSI $(n=67)$ & VRE BSI $(n=24)$ & $p$-value \\
\hline Age, years & $48.0 \pm 14.0$ & $44.9 \pm 14.5$ & 0.356 \\
\hline Male sex & $41(61.2)$ & $10(41.7)$ & 0.098 \\
\hline Underlying hematologic diseases & & & 0.046 \\
\hline AML & $41(61.2)$ & $10(41.7)$ & 0.098 \\
\hline ALL & $18(26.9)$ & $13(54.2)$ & 0.015 \\
\hline Others $^{a}$ & $8(11.9)$ & $1(4.2)$ & 0.436 \\
\hline Treatment for underlying diseases & & & 0.861 \\
\hline Chemotherapy & $56(83.6)$ & $20(83.3)$ & $>0.999$ \\
\hline Allogeneic SCT & $9(13.4)$ & $4(16.7)$ & 0.738 \\
\hline Autologous SCT & $2(3.0)$ & $0(0)$ & $>0.999$ \\
\hline Enterococcal species & & & $<0.001$ \\
\hline E. faecium & $44(65.7)$ & $22(91.7)$ & 0.016 \\
\hline E. faecalis & $23(34.3)$ & $0(0.0)$ & $<0.001$ \\
\hline E. gallinarum & $0(0.0)$ & $2(8.3)$ & 0.067 \\
\hline SAPS $\|$ at the onset of BSI & 39 (28-95) & $41(31-74)$ & 0.336 \\
\hline Charlson's comorbidity index & $2(0-4)$ & $2(0-4)$ & 0.644 \\
\hline ICU care & $16(23.9)$ & $7(29.2)$ & 0.609 \\
\hline Septic shock & $8(11.9)$ & $2(8.3)$ & $>0.999$ \\
\hline Length of hospitalization, day & $22(13-44)$ & $26(17-77)$ & 0.025 \\
\hline Duration of neutropenia before BSI, day & $14(0-156)$ & $15(6-27)$ & 0.908 \\
\hline Severity of neutropenia & & & 0.112 \\
\hline ANC $<500 / \mathrm{mm}^{3}$ & $2(3.0)$ & $3(12.5)$ & \\
\hline ANC $<100 / \mathrm{mm}^{3}$ & $65(97.0)$ & $21(87.5)$ & \\
\hline Administration of adequate antibiotics & & & 0.002 \\
\hline$\leq 48$ hours after febrile episode & $50(75.8)$ & $10(41.7)$ & \\
\hline$>48$ hours after febrile episode & $16(24.2)$ & $14(58.3)$ & \\
\hline
\end{tabular}

Data are presented as $\mathrm{n}(\%)$, mean \pm SD or median (range). Abbreviations: $A L L$ acute lymphoblastic leukemia, $A M L$ acute myeloid leukemia, $A N C$ absolute neutrophil count, BSI bloodstream infection, ICU intensive care unit, SAPS /I Simplified Acute Physiology Score, SCT stem cell transplantation, VRE, vancomycin-resistant enterococci, VSE vancomycin-susceptible enterococci. ${ }^{a}$ Myelodysplastic syndrome $(n=2)$, multiple myeloma $(n=1)$, aplastic anemia $(n=3)$, lymphoma $(n=2)$ and hemophagocytic lymphohistiocytosis $(n=1)$.

E. faecium isolates, all were resistant to ampicillin, $75.8 \%$ (50 of 66) showed high-level resistance to gentamicin, and all were susceptible to quinupristin-dalfopristin. Two $E$. faecium isolates were not susceptible to linezolid: one isolate showed intermediate susceptibility and one showed resistance; in both, the MIC changed to susceptible when the blood cultures were repeated. Among the E. faecalis isolates, $69.6 \%$ (16 of 23) were resistant to high-level gentamicin, and all isolates were susceptible to glycopeptides and linezolid. For tigecycline, 97.0\% (64 of 66) of the $E$. faecium and $95.7 \%$ (22 of 23) of the E. faecalis isolates were susceptible.

Among the 24 VRE BSI patients, rectal swabs for VRE were performed in 20 patients, $90 \%$ (18 of 20) of whom

Table 2 Outcomes of enterococcal bloodstream infection in the VSE and VRE BSI groups

\begin{tabular}{|c|c|c|c|c|}
\hline & Total $(n=91)$ & VSE BSI $(n=67)$ & VRE BSI $(n=24)$ & $P$-value \\
\hline 7-day clinical treatment failure & $30(33.0)$ & $18(26.9)$ & $12(50.0)$ & 0.040 \\
\hline 7-day microbiologic failure & $12(13.2)$ & $8(11.9)$ & $4(16.7)$ & 0.499 \\
\hline \multicolumn{5}{|l|}{ Mortality } \\
\hline Attributable mortality & $10(11.0)$ & $6(9.0)$ & $4(16.7)$ & 0.447 \\
\hline Crude mortality & $21(23.9)$ & $15(22.7)$ & $6(27.3)$ & 0.059 \\
\hline
\end{tabular}

Data are presented as $\mathrm{n}(\%)$. Abbreviations: BSI bloodstream infection, VRE vancomycin-resistant enterococci, VSE vancomycin-susceptible enterococci. 


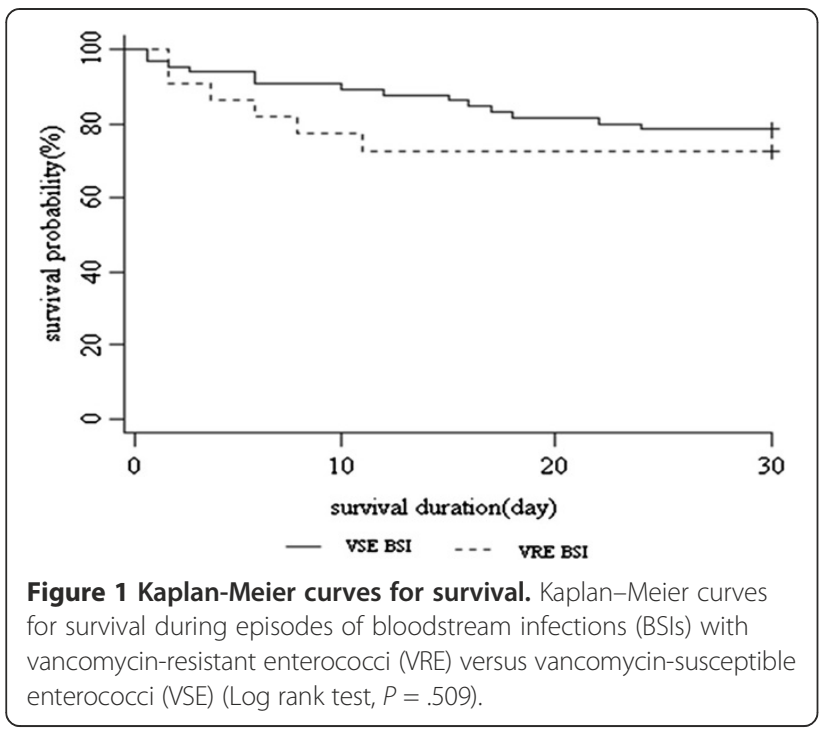

Table 3 Factors associated with 30-day mortality in patients with enterococcal bloodstream infections

\begin{tabular}{|c|c|c|}
\hline & $\begin{array}{l}\text { Unadjusted HR } \\
\quad(95 \% \mathrm{Cl})\end{array}$ & $\begin{array}{c}\text { Adjusted HR } \\
(95 \% \mathrm{Cl})\end{array}$ \\
\hline Age, years & $1.03(0.99-1.06)$ & \\
\hline Male sex & $1.00(0.62-1.62)$ & \\
\hline Vancomycin resistance & $1.38(0.53-3.59)$ & $0.75(0.24-2.36)$ \\
\hline $\begin{array}{l}\text { SAPS II at the onset of BSI } \\
\text { (per } 1 \text { point) }\end{array}$ & $1.12(1.08-1.17)$ & $1.13(1.08-1.18)$ \\
\hline \multicolumn{3}{|l|}{ Underlying hematologic diseases } \\
\hline AML & 1.00 & \\
\hline ALL & $0.78(0.27-2.24)$ & \\
\hline Others & $2.29(0.73-7.19)$ & \\
\hline \multicolumn{3}{|l|}{ Treatment for underlying diseases } \\
\hline Chemotherapy & 1.00 & \\
\hline SCT & $1.33(0.72-2.47)$ & \\
\hline Length of hospitalization, day & $1.05(1.02-1.09)$ & $1.00(0.98-1.02)$ \\
\hline $\begin{array}{l}\text { Duration of neutropenia before BSI, } \\
\text { day }\end{array}$ & $1.00(0.98-1.02)$ & \\
\hline \multicolumn{3}{|l|}{ Severity of neutropenia } \\
\hline ANC $\left(<500 / \mathrm{mm}^{3}\right)$ & 1.00 & \\
\hline $\operatorname{ANC}\left(<100 / \mathrm{mm}^{3}\right)$ & $0.43(0.10-1.86)$ & \\
\hline $\begin{array}{l}\text { Delayed administration of adequate } \\
\text { antibiotics ( }>48 \text { hours) }\end{array}$ & $0.78(0.30-2.04)$ & \\
\hline
\end{tabular}

Abbreviations: ALL acute lymphoblastic leukemia, $A M L$ acute myeloid leukemia, $A N C$ absolute neutrophil count, $B S I$ bloodstream infection, $C l$ Confidence interval, HR Hazard ratio, ICU intensive care unit, SAPS // Simplified Acute Physiology Score, SCT stem cell transplantation, OR Odds ratio, VRE vancomycin-resistant enterococci, VSE vancomycin-susceptible enterococci.
Table 4 In vitro susceptible rate according to Enterococcus species

\begin{tabular}{lccc}
\hline & $\begin{array}{c}\text { E. faecium } \\
(\mathbf{n}=66)\end{array}$ & $\begin{array}{c}\text { E. faecalis } \\
(\mathbf{n}=\mathbf{2 3})\end{array}$ & $\begin{array}{c}\text { E. gallinarum } \\
(\mathbf{n}=\mathbf{2})\end{array}$ \\
\hline Ampicillin & $0(0)$ & - & $2(100)$ \\
High level gentamicin synergy & $16(24.24)$ & $7(30.43)$ & $2(100)$ \\
Vancomycin & $44(66.67)$ & $23(100)$ & $0(0)$ \\
Teicoplanin & $47(71.21)$ & $23(100)$ & $2(100)$ \\
Linezolid & $64(96.97)$ & $23(100)$ & $2(100)$ \\
Quinupristin-dalfopristin & $66(100)$ & $0(0)$ & $0(0)$ \\
Tigecycline & $64(98.46)$ & $22(95.65)$ & $2(100)$ \\
\hline Data are presented as $\mathrm{n}(\%)$. & & &
\end{tabular}

were positive. Results of rectal swabs done previous to diagnosis of VRE BSI were only available in 9 patients, 2 of whom (22.2\%) showed a positive result, indicating rectal VRE colonization prior to BSI.

We conducted a subgroup analysis of the $66 \mathrm{E}$. faecium BSIs to eliminate the differences that may have resulted from inclusion of multiple enterococcal species in the overall study cohort. This analysis showed concordant mortality results between vancomycin-susceptible and vancomycin-resistant E. faecium.

\section{Discussion}

This study examined the impact of vancomycin resistance on mortality in neutropenic patients with enterococcal BSI. The results in this study indicate that vancomycin resistance does not affect the 7-day and 30-day mortalities in a relatively homogeneous group of patients. A meta-analysis estimated that VRE BSI is associated with a 2.52 -fold increased mortality [1]. However, it is still controversial whether VRE BSI results in higher mortality than VSE BSI in neutropenic patients $[12,18,19]$. In general, the clinical impact of vancomycin resistance is thought to be more pronounced in immunocompromised patients. Several reports indicate that hematological malignancy is a risk factor for VRE infections $[7,8]$. However, the use of antibiotics and severity of underlying disease should be considered in any outcome analysis because outcomes may be affected by variables such as antibiotic treatment strategies during treatment for neutropenic fever.

There was a significant delay in effective anti-VRE therapy for over 48 hours, and the maximum delay was 5 days based on the culture results in the VRE BSI group. However, despite the delayed administration of adequate antibiotics and delayed improvement of clinical signs of SIRS in the VRE group, the 7- and 30-day mortality rates did not differ between the two groups. One report indicates that VRE is a dominant pathogen before engraftment in the setting of SCT, emphasizing the potential utility of the empirical treatment of Gram-positive 
organisms in the pre-engraftment period in febrile SCT patients [14]. But, there are many considerations to decide empirical antibiotics including incidence, risk and outcomes. In addition, virulence of the pathogen, breakthrough infection, overtreatment and emerging resistance are also important in decision making. Our results suggest that clinicians need to be careful to add anti-VRE therapy empirically or pre-emptively before obtaining the final microbiology results. Current antibiotics strategy of adding glycopeptides in febrile neutropenic patients with presumptive Gram-positive bacteremia might be sufficient on the premise that treatment must be individualized.

Mortality related to VRE BSI has been reported to vary from 4 80\% [9-18,27,28]. As mentioned above, such a broad range may stem from the diverse underlying conditions and different antimicrobial strategies. Recently, Vydra et al. reported that mortality 30 days after enterococcal infection was 38\% for both VRE and VSE BSI cases in SCT patients and non-relapse mortality 1 year after transplant was 53\% and 33\% for VRE and VSE BSI cases respectively [27]. These results are similar to ours, as the 30-day mortality showed no difference between the two groups, while the 1-year mortality after SCT in VRE infections may reflect the severity of the underlying medical condition, rather than the poor outcome portended by vancomycin resistance itself. Interestingly, higher vancomycin resistance rate was reported in their cohort, with 66\% VRE BSI among enterococcal BSI compared to ours. In their cohort, standard antibiotic therapy included ceftazidime, vancomycin, and often tobramycin until results of cultures were obtained, and such a strategy may have influenced the vancomycin resistance rate in enterococcal BSIs.

Possible causes that our results showed no difference between the VSE and VRE BSI group include the following; first, the known higher mortality in the VRE BSI group may be due to host factors, rather than a pathogen-related factor [5,19]. Our study population was a relatively homogeneous group that showed neutropenia after treatment for hematological malignancies. The statistically equivalent initial SAPS II at the onset of BSI may have contributed to the similar outcome of the two groups. Second, the differences in virulence amongst the enterococcal species can be considered. E. faecium is more frequently associated with vancomycin resistance than is E. faecalis. Some studies have examined animal models, with one study using an intestinal model of Caenorhabditis elegans reporting that low inoculums of E. faecalis grow to a high titer in the C. elegans intestine, resulting in persistent infection and death, whereas a high ingested titer of E. faecium also accumulates in the nematode gut, but did not cause death [29]. Another animal study reported that in an intraperitoneal mouse model, a greater inoculum size was required with E. faecium than with E. faecalis strains [30]. Based on these results, the higher virulence of $E$. faecalis species may have impacted upon the similar mortality shown in the VSE and VRE groups.

Interestingly, a difference in the vancomycin resistance rate between myeloid and lymphoid malignancy was observed, with more patients with ALL in the VRE group. There are some reports that neutrophils play a critical role in the pathogenesis of enterococcal infections [31,32]. However, the immune responses to Enterococci are poorly understood. Our finding is not consistent with the results of Worth et al., who identified an underlying diagnosis of AML as a risk factor for VRE [33]. Theirs was a case-control study between patients with and without VRE infection, not between VRE- and VSE-infected groups. Moreover, there was an outbreak of VRE infection in the hematology unit, and empirical antibiotic therapy was modified during the study period. These factors may explain discrepancies found between their results and ours, although further studies are needed.

According to CDC criteria for nosocomial infection, enterococcal BSI was defined as any blood culture growing enterococcus species [34]. But diverse definitions have been used for each study ( $\geq 1$ or 2 blood culture results), which may result in selection bias. Therefore, to use a strict BSI definition, we included cases with two or more positive blood cultures of enterococcal species $[1,8]$. We also included only BSI cases that occurred during neutropenia related to their treatment with exclusion of cases undergone palliative care. This study design made our study population as a relatively homogeneous group with reversible neutropenia. And we calculated the incidence rate of enterococcal BSI considering hospital stay. In addition, a subgroup analysis was performed for E. faecium BSI, and gave concordant results. Our investigations have important implications for clinical practice.

Nevertheless, our study has some limitations. First, it was a retrospective study. The retrospective design made it difficult to evaluate the duration of bacteremia and gastrointestinal disease. Second, VRE surveillance is not routinely performed in our center, data for VRE colonization would be insufficient $[22,35]$. Third, we selected the definition of enterococcal BSI with 2 or more blood culture positivity, the incidence rate might be affected. Fourth, it had a limitation stemming from its sample size. Based on a previous meta-analysis [1], we assumed a crude mortality for the VSE BSI group of $20 \%$ and an odds ratio (OR) for vancomycin resistance on mortality of 2.52. Based on these assumptions, the statistical power of this study to detect a significant difference in mortality is a power of $40 \%$ in a 2 -sided test with a probability of 0.05 . However the mortality data and the OR 
to calculate the sample sizes are not for an immunocompromised population and may include confounding effects of underlying diseases and severity.

\section{Conclusions}

In summary, our data suggest that enterococcal BSI due to vancomycin-resistant species in patients with hematological malignancies tends to occur after a long period of hospitalization. SAPS-II predicts poor outcome, reflecting patient age and the severity of illness. By contrast, severe neutropenia and vancomycin resistance itself did not appear to affect the fatality rate. The attributable and crude mortality were not different between VRE and VSE BSI groups despite the delayed use of adequate antibiotics in a relatively homogeneous immunocompromised group. Further prospective controlled studies are needed.

\section{Competing interests}

The authors declare that they have no competing interests.

\section{Authors' contributions}

SYC conducted the hospital chart review and analysis of resulting data, interpreted data, drafted the initial study report and wrote the final report. DGL conceptualized the study and formulated the study design, and contributed to data interpretation. He revised and edited the manuscript. SMC participated in critical revision of manuscript. JCK, SHK, SHP, JHC and $\mathrm{JHY}$ contributed the data interpretation and editing the manuscript. JKC participated in data collection and study logistics. YJP contributed the interpretation of microbiologic data. All authors read and approved the final manuscript.

\section{Acknowledgements}

The statistical consultation was supported by Catholic Research Coordinating Center of the Korea Health 21 R\&D Project (A070001), Ministry of Health \& Welfare, Republic of Korea. Further, we would like to thank Jae Wook Lee, Department of Pediatrics, The catholic University of Korea (Seoul, Korea) for English proofreading.

\section{Financial disclosure}

There was no financial support for this study.

\section{Previous presentation}

Part of this study was presented at the $52^{\text {nd }}$ Interscience Conference on Antimicrobial Agents and Chemotherapy in San Francisco, September 9-12, 2012 (K-1632).

\section{Author details}

${ }^{1}$ Division of Infectious Diseases, Department of Internal Medicine, Vaccine Bio Research Institute, College of Medicine, The Catholic University of Korea, Seoul, Republic of Korea. ${ }^{2}$ The Catholic Blood and Marrow Transplantation Center, Seoul St. Mary's Hospital, College of Medicine, The Catholic University of Korea, Seoul, Republic of Korea. ${ }^{3}$ Department of Internal Medicine, Daniel Hospital, GyeongGi-Do, Republic of Korea. ${ }^{4}$ Department of Life Sciences, Pohang University of Science and Technology, Pohang, Republic of Korea. ${ }^{5}$ Department of Laboratory Medicine, College of Medicine, The Catholic University of Korea, Seoul, Republic of Korea.

Received: 31 March 2013 Accepted: 22 October 2013 Published: 29 October 2013

\section{References}

1. DiazGranados CA, Zimmer SM, Klein M, Jernigan JA: Comparison of mortality associated with vancomycin-resistant and vancomycinsusceptible enterococcal bloodstream infections: a meta-analysis. Clin Infect Dis 2005, 41:327-333.
2. Bonten $M J$, Willems R, Weinstein RA: Vancomycin-resistant enterococci: Why are they here, and where do they come from? Lancet Infect Dis 2001, 1:314-325.

3. Moellering RC Jr: Emergence of Enterococcus as a significant pathogen. Clin Infect Dis 1992, 14:1173-1176.

4. Kwon JC, Kim SH, Choi JK, Cho SY, Park YJ, Park SH, Choi SM, Lee DG, Choi JH, Yoo JH: Epidemiology and clinical features of bloodstream infections in hematological wards: one year experience at the catholic blood and marrow transplantation center. Infect Chemother 2013, 45:51-61.

5. McKinnell JA, Patel M, Shirley RM, Kunz DF, Moser SA, Baddley JW: Observational study of the epidemiology and outcomes of vancomycinresistant Enterococcus bacteraemia treated with newer antimicrobial agents. Epidemiol Infect 2011, 139:1342-1350.

6. Han SH, Chin BS, Lee HS, Jeong SJ, Choi HK, Kim CO, Yong D, Choi JY, Song YG, Lee K, Kim JM: Vancomycin-resistant enterococci bacteremia: risk factors for mortality and influence of antimicrobial therapy on clinical outcome. J Infect 2009, 58:182-190.

7. Shay DK, Maloney SA, Montecalvo M, Banerjee S, Wormser GP, Arduino MJ, Bland LA, Jarvis WR: Epidemiology and mortality risk of vancomycin-resistant enterococcal bloodstream infections. J Infect Dis 1995, 172:993-1000.

8. Vergis EN, Hayden MK, Chow JW, Snydman DR, Zervos MJ, Linden PK, Wagener MM, Schmitt B, Muder RR: Determinants of vancomycin resistance and mortality rates in enterococcal bacteremia. A prospective multicenter study. Ann Intern Med 2001, 135:484-492.

9. Edmond MB, Ober JF, Dawson JD, Weinbaum DL, Wenzel RP: Vancomycinresistant enterococcal bacteremia: natural history and attributable mortality. Clin Infect Dis 1996, 23:1234-1239.

10. Bhavnani SM, Drake JA, Forrest A, Deinhart JA, Jones RN, Biedenbach DJ, Ballow CH: A nationwide, multicenter, case-control study comparing risk factors, treatment, and outcome for vancomycin-resistant and -susceptible enterococcal bacteremia. Diagn Microbiol Infect Dis 2000, 36:145-158.

11. Lodise TP, McKinnon PS, Tam VH, Rybak MJ: Clinical outcomes for patients with bacteremia caused by vancomycin-resistant enterococcus in a level 1 trauma center. Clin Infect Dis 2002, 34:922-929.

12. DiazGranados CA, Jernigan JA: Impact of vancomycin resistance on mortality among patients with neutropenia and enterococcal bloodstream infection. J Infect Dis 2005, 191:588-595.

13. Linden PK, Pasculle AW, Manez R, Kramer DJ, Fung JJ, Pinna AD, Kusne S: Differences in outcomes for patients with bacteremia due to vancomycin-resistant Enterococcus faecium or vancomycin-susceptible E. faecium. Clin Infect Dis 1996, 22:663-670.

14. Kamboj M, Chung D, Seo SK, Pamer EG, Sepkowitz KA, Jakubowski AA, Papanicolaou G: The changing epidemiology of vancomycin-resistant Enterococcus (VRE) bacteremia in allogeneic hematopoietic stem cell transplant (HSCT) recipients. Biol Blood Marrow Transplant 2010, 16:1576-1581.

15. Lautenbach E, Bilker WB, Brennan PJ: Enterococcal bacteremia: risk factors for vancomycin resistance and predictors of mortality. Infect Control Hosp Epidemiol 1999, 20:318-323.

16. Lucas GM, Lechtzin N, Puryear DW, Yau LL, Flexner CW, Moore RD: Vancomycinresistant and vancomycin-susceptible enterococcal bacteremia: comparison of clinical features and outcomes. Clin Infect Dis 1998, 26:1127-1133.

17. Garbutt JM, Ventrapragada M, Littenberg B, Mundy LM: Association between resistance to vancomycin and death in cases of Enterococcus faecium bacteremia. Clin Infect Dis 2000, 30:466-472.

18. Yoo JH, Lee DG, Choi SM, Choi JH, Shin WS, Kim M, Yong D, Lee K, Min WS, Kim CC: Vancomycin-resistant enterococcal bacteremia in a hematology unit: molecular epidemiology and analysis of clinical course. J Korean Med Sci 2005, 20:169-176.

19. Todeschini G, Tecchio C, Borghero C, D'Emilio A, Pegoraro E, de Lalla F, Benedetti P, Spolaore P, Pellizzer G: Association between Enterococcus bacteraemia and death in neutropenic patients with haematological malignancies. J Infect 2006, 53:266-273.

20. Lee DG, Kim SH, Kim SY, Kim C, Park WB, Song YG, Choi JH: Evidence-based guidelines for empirical therapy of neutropenic fever in Korea. Korean J Intern Med 2011, 26:220-252.

21. Weissinger F, Auner HW, Bertz H, Buchheidt D, Cornely OA, Egerer G, Heinz W, Karthaus M, Kiehl M, Krüger W, Penack O, Reuter S, Ruhnke M, Sandherr M, Salwender HJ, Ullmann AJ, Waldschmidt DT, Wolf HH: Antimicrobial therapy of febrile complications after high-dose chemotherapy and autologous hematopoietic stem cell transplantation-guidelines of the infectious diseases working party (AGIHO) of the German society of hematology and oncology (DGHO). Ann Hematol 2012, 91:1161-1174. 
22. Tomblyn M, Chiller T, Einsele H, Gress R, Sepkowitz K, Storek J, Wingard JR, Young JA, Boeckh MJ: Guidelines for preventing infectious complications among hematopoietic cell transplant recipients: a global perspective. Biol Blood Marrow Transplant 2009, 15:1143-1238.

23. Freifeld AG, Bow EJ, Sepkowitz KA, Boeckh MJ, Ito Jl, Mullen CA, Raad II, Rolston KV, Young JA, Wingard JR, Infectious Diseases Society of America: Clinical practice guidelines for the use of antimicrobial agents in neutropenic patients with cancer: 2010 update by the infectious diseases society of America. Clin Infect Dis 2011, 52:e56-e93.

24. Lee DG, Yim DS, Choi SM, Park SH, Yoo JH, Choi JH, Min WS, Shin WS, Kim CC: Efficacies of teicoplanin in patients with febrile neutropenia. Infect Chemother 2004, 36:83-91.

25. Ortega M, Rovira M, Almela M, Marco F, de la Bellacasa JP, Martínez JA, Carreras E, Mensa J: Bacterial and fungal bloodstream isolates from 796 hematopoietic stem cell transplant recipients between 1991 and 2000. Ann Hematol 2005, 84:40-46.

26. Kim SH, Kwon JC, Choi SM, Lee DG, Park SH, Choi JH, Yoo JH, Cho BS, Eom KS, Kim YJ, Kim HJ, Lee S, Min CK, Cho SG, Kim DW, Lee JW, Min WS: Escherichia coli and Klebsiella pneumoniae bacteremia in patients with neutropenic fever: factors associated with extended-spectrum $\beta$-lactamase production and its impact on outcome. Ann Hematol 2013, 92:533-541.

27. Vydra J, Shanley RM, George I, Ustun C, Smith AR, Weisdorf DJ, Young JH: Enterococcal bacteremia is associated with increased risk of mortality in recipients of allogeneic hematopoietic stem cell transplantation. Clin Infect Dis 2012, 55:764-770.

28. Peel T, Cheng AC, Spelman T, Huysmans M, Spelman D: Differing risk factors for vancomycin-resistance and vancomycin-sensitive enterococcal bacteraemia. Clin Microbiol Infect 2012, 18:388-394.

29. Garsin DA, Sifri CD, Mylonakis E, Qin X, Singh KV, Murray BE, Calderwood SB, Ausubel FM: A simple model host for identifying Gram-positive virulence factors. Proc Natl Acad Sci USA 2001, 98:10892-10897.

30. Nannini EC: Vancomycin-resistant enterococcal bloodstream infectionrelated mortality: focus on the lack of appropriate therapy. Clin Infect Dis 2006, 42:1203-1204

31. Rakita RM, Vanek NN, Jacques-Palaz K, Mee M, Mariscalco MM, Dunny GM, Snuggs M, Van Winkle WB, Simon SI: Enterococcus faecalis bearing aggregation substance is resistant to killing by human neutrophils despite phagocytosis and neutrophil activation. Infect Immun 1999, 67:6067-6075

32. Arduino RC, Jaques-Palaz K, Murray BE, Rakita RM: Resistance of Enterococcus faecium to neutrophil-mediated phagocytosis. Infect Immun 1994, 62:5587-5594.

33. Worth $L$, Thursky KA, Seymour JF, Slavin MA: Vancomycin-resistant Enterococcus faecium infection in patients with hematologic malignancy: patients with acute myeloid leukemia are at high-risk. Eur J Haematol 2007, 79:226-233.

34. Garner JS, Jarvis WR, Emori TG, Horan TC, Hughes JM: CDC definitions for nosocomial infections, 1988. Am J Infect Control 1988, 16:128-140.

35. Weinstock DM, Conlon M, lovino D, Aubrey T, Gudiol C, Riedel E, Young JW, Kiehn TE, Zuccotti G: Colonization, bloodstream infection, and mortality caused by vancomycin-resistant enterococcus early after allogeneic hematopoietic stem cell transplant. Biol Blood Marrow Transplant 2007, 13:615-621.

doi:10.1186/1471-2334-13-504

Cite this article as: Cho et al:: Impact of vancomycin resistance on mortality in neutropenic patients with enterococcal bloodstream infection: a retrospective study. BMC Infectious Diseases 2013 13:504.

\section{Submit your next manuscript to BioMed Central and take full advantage of:}

- Convenient online submission

- Thorough peer review

- No space constraints or color figure charges

- Immediate publication on acceptance

- Inclusion in PubMed, CAS, Scopus and Google Scholar

- Research which is freely available for redistribution

Submit your manuscript at www.biomedcentral.com/submit 